Doug Geisler, Eva K. Grebel, and Dante Minniti, eds.

\title{
Formation of Twin Clusters in a Galactic Tidal Field
}

\author{
Christian Theis
}

Institut f. Theoretische Physik und Astrophysik, Universität Kiel, 24098 Kiel, Germany, e-mail: theis@astrophysik.uni-kiel.de

\begin{abstract}
The formation of globular clusters is still an unsolved problem. Though most scenarios assume a massive molecular cloud as the progenitor, it is unclear how the cloud is transformed into a star cluster. Here a scheme of supernova (SN) induced cluster formation is investigated. In this scenario the expanding SN shell accumulates the mass of the cloud. This is accompanied by fragmentation resulting in star formation in the shell. If this stellar shell expands sufficiently slowly, its self-gravity leads to a recollapsing shell, thus forming one or several stellar clusters.

In this paper N-body simulations of collapsing shells moving on circular orbits in a galactic potential are presented. It is shown that typical shells $\left(10^{5} \mathrm{M}_{\odot}, 30 \mathrm{pc}\right)$ evolve to twin clusters in the galactocentric distance range between 3 and $11 \mathrm{kpc}$. Their masses show a strong radial trend: on orbits inside $5 \mathrm{kpc}$ both clusters have almost equal mass. Outside $5 \mathrm{kpc}$ the more massive twin cluster contains about $55 \%$ of the shell's mass, whereas the mass of the smaller decreases linearily to $15 \%$ at 11 $\mathrm{kpc}$. Outside $11 \mathrm{kpc}$ the collapsing shells end up in a single cluster. Inside $3 \mathrm{kpc}$ the shells are tidally disrupted and only fragments substantially less massive than the initial shell survive.
\end{abstract}

\section{Introduction}

Several scenarios for the formation of globular cluster have been suggested, e.g. the collapse of giant molecular clouds (GMC) or the collision of molecular clouds (e.g. Fall \& Rees 1985; Murray \& Lin 1990; Fujimoto \& Kumai 1997). A common characteristic of all these scenarios is that the clusters are formed from smooth gaseous distributions which are transformed into stars. However, this assumption requires short formation timescales and unusually high star formation efficiencies in order to end up with a gravitationally bound cluster. An alternative model suggested by Brown et al. (1991) can overcome these difficulities: their scenario starts with an OB-association exploding near the center of a molecular cloud. The expanding shell sweeps up the cloud material and in a later stage the expansion can be decelerated or stopped by both the accumulated mass and the external pressure of the ambient hot gas. The shell itself undergoes fragmentation and, finally, forms stars. If these stars are gravitationally bound, they will recollapse, forming a globular cluster.

Though a discrimination between the scenarios by means of hydrodynamical simulations (starting from first principles) is far out of reach at the moment, one can study different evolutionary stages. E.g., Theis (2001) compared in a 

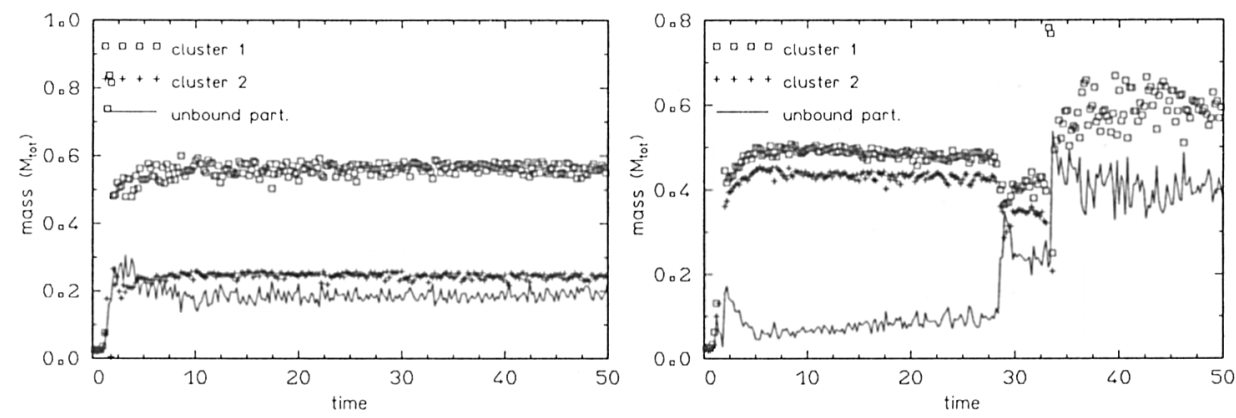

Figure 1. Temporal evolution of the masses of the twin clusters (signs) and the mass of unbound particles (solid line). Shown are results for circular orbits at a galactocentric distance of $10 \mathrm{kpc}$ (left) and $5 \mathrm{kpc}$ (right). The twins in the right panel collide at $t \approx 28.6$ and merge at $t \approx 33.4$. The total mass in each simulation is $10^{5} \mathrm{M}_{\odot}$. The time unit is $7.7 \mathrm{Myr}$.

series of N-body simulations the collapse of thin stellar shells and homogeneous spheres in a galactic tidal field. These calculations were performed for circular and eccentric orbits, but with a constant apogalacticon of $5 \mathrm{kpc}$. It was found that collapsing shells preferably end in multiple systems, mainly twins, whereas homogeneous spheres either form single clusters or become completely disrupted. The twins might survive for - at least - several galactic revolutions, and some of them show kinematical peculiarities like counter-rotating cores.

In this paper I will address the question of how the formation of twins depends on the galactocentric distance, i.e., the strength of the galactic tidal field.

\section{Numerical Models}

The simulations start with a thin, spherical shell of a mass $\mathrm{M}_{\text {tot }}=10^{5} \mathrm{M}_{\odot}$, an outer radius of $30 \mathrm{pc}$ and a thickness of $3 \mathrm{pc}$. The shell is initially at rest in the sense that there is no overall expansion or contraction of the shell with respect to its center. The potential of the Galaxy is modelled by an isothermal halo with a circular speed of $220 \mathrm{~km} \mathrm{~s}^{-1}$. In all simulations the initial velocity of the shell corresponds to a circular orbit. The calculations are performed with $N=10^{4}$ particles using a GRAPE3 board.

Fig. 1 shows the temporal evolution of the masses of the two largest clusters for galacatocentric radii of 5 and $10 \mathrm{kpc}$. At $10 \mathrm{kpc}$ two clusters with a mass ratio of 5:2 are formed after 20 Myr. Both clusters survive until the end of the simulation $(\sim 400 \mathrm{Myr})$. Twenty percent of the stars initially residing in the shell became unbound. A slightly different behaviour is seen for the model starting at $5 \mathrm{kpc}$ : Again twins are formed, but they have almost equal mass. After 210 Myr they collide and, finally, they merge. Both events are clearly reflected in step-like increases of the number of unbound particles which amounts finally to $40 \%$ of the mass of the shell (Fig. 1, right). 

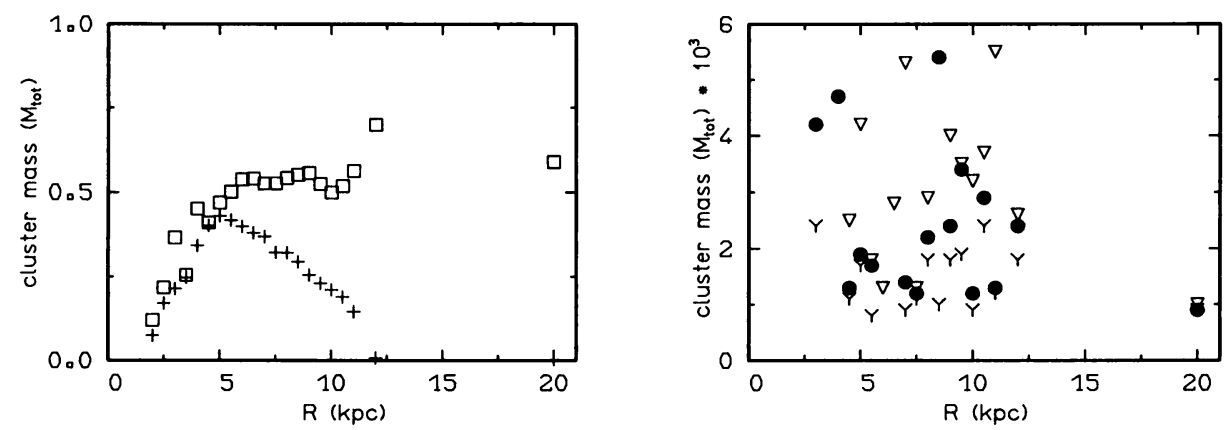

Figure 2. Cluster mass vs. galactocentric distance. Shown are the masses of the five most massive clusters of each simulation $18.6 \mathrm{Myr}$ after the collapse. The distribution is unaffected by later merging of clusters. The clusters are sorted by mass (left: most massive clusters (box, plus), right: smaller clusters (triangle, filled circle, Y)).

The masses of the formed clusters exhibit a clear radial trend (Fig. 2): The mass of the largest cluster increases almost linearily from 2 to $5.5 \mathrm{kpc}$ reaching a plateau of about $55 \%$ of the total mass. Beyond $11 \mathrm{kpc}$ only one massive cluster is formed. About $40 \%$ of the stars are not bound in clusters. Inside $3 \mathrm{kpc}$, a large set of small clusters is formed instead of a dominating pair of clusters. The mass ratio $q$ between the two most massive clusters also shows two regimes: Below $5 \mathrm{kpc}$ the masses are almost identical, whereas outside $5 \mathrm{kpc} q$ increases up to $\sim 4: 1$ at $11 \mathrm{kpc}$. In addition to the large clusters $\left(>10^{4} \mathrm{M}_{\odot}\right)$, typically several small $\left(<10^{3} \mathrm{M}_{\odot}\right)$, gravitationally bound clusters are formed. Contrary to the large clusters, their mass shows no trends with galactocentric distance.

The simulations demonstrate that twin formation is expected over a large radial range. About $1 / 3$ of these twins merge within $400 \mathrm{Myr}$ after their formation. The surviving twins are characterized by large spatial separations which makes them less likely to undergo a subsequent merger. Therefore, twin globulars might still exist in the Milky Way, but they cannot be identified as twins due to their large separation. Since they should share common orbits and metallicities, they could be found by proper determinations of globular cluster orbits.

Acknowledgments. The author is grateful to the Deutsche Forschungsgemeinschaft (DFG) for the travel support under grant TH511/3-1. The analysis of the cluster sizes has been performed with the SKID program kindly made available by the NASA HPCC ESS group at the University of Washington.

\section{References}

Brown, J.H., Burkert, A., \& Truran, J.W. 1991, ApJ, 376, 115

Fall, S.M., Rees, M.J. 1985, ApJ, 298, 18

Fujimoto, M., Kumai, Y. 1997, AJ, 113, 249

Murray, S.D., Lin, D.N.C. 1990, ApJ, 363, 50

Theis, Ch. 2001, in Dynamics of Star Clusters and the Milky Way, ASP Conf. Ser. 228, eds. S. Deiters et al., (Provo: ASP), 347 